\title{
Strategic Delay: Histology- and Biology-Driven Decision-Making in Recurrent Retroperitoneal Sarcoma
}

\author{
Carol J. Swallow, MD, PhD, FRCS(C), FACS ${ }^{1,2}$ \\ ${ }^{1}$ Division of General Surgery, University of Toronto, Toronto, Canada; ${ }^{2}$ Department of Surgical Oncology, Princess \\ Margaret Cancer Centre/Mount Sinai Hospital, Toronto, Ontario, Canada
}

In this issue, the sarcoma group at the MD Anderson Cancer Center (MDACC) has tackled the important and challenging problem of well-differentiated liposarcoma (WD-LPS) of the retroperitoneum, as part of a series of recent contributions that explore approaches to retroperitoneal sarcoma (RPS) and outcomes at their institution. ${ }^{1}$ In particular, Ikoma et al. report the survival and re-recurrence rates after resection of recurrent WD-LPS of the retroperitoneum. Their results are a stark reminder of the serious impact that the diagnosis of primary RP WD-LPS has on patients' expectations for life and of the gravity of recurrence.

Retroperitoneal LPS is subclassified into four distinct histologic types: (1) well-differentiated (WD), (2) dedifferentiated (DD), (3) myxoid, and (4) round cell; the latter two account for only $5-10 \%$ of cases. Pleomorphic LPS is extremely rare in the retroperitoneum, and most large series do not include any cases. In most series of primary RPS, DD and WD comprise about an equal share of the cases. There is a prevalent belief that WD-LPS of the RP can develop into DD-LPS over time and with serial recurrences, but it is not altogether clear whether the emergence of a dedifferentiated component reflects what was the true nature of the tumor all along. It is important to note that the entity once known as MFH of the retroperitoneum is now considered to represent DD-LPS, a reclassification that has been facilitated by molecular subtyping (immunohistochemistry and FISH positivity for amplification of mdm2).

\section{(C) Society of Surgical Oncology 2018}

First Received: 20 March 2018;

Published Online: 4 June 2018

C. J. Swallow, MD, PhD, FRCS(C), FACS

e-mail: Carol.Swallow@sinaihealthsystem.ca
The distinction between atypical lipomatous tumor (ALT) of the extremity and WD-LPS of the retroperitoneum bears emphasis. While the two entities appear histologically similar, the implications for disease-free survival (DFS), quality of life, and even overall survival (OS) are quite different. In the majority of cases, extremity ALT is appropriately managed with resection alone, with no risk of distant recurrence and a low risk of local recurrence. Most authors agree that WD-LPS arising in the retroperitoneum does not give rise to distant metastases. For instance, the distant metastasis-free survival (DMFS) at 3 years was $99 \%$ in 99 patients with this diagnosis operated on at MSKCC. ${ }^{2}$ This was in contradistinction to DD-LPS $(N=65)$, which had a 70\% DMFS at 3 years. However, the risk of local (i.e., ipsilateral retroperitoneum and/or peritoneal cavity and/or pelvis) failure following resection of primary WD-LPS of the retroperitoneum is reported to range from 20 to $50 \%$ at 5 years. ${ }^{2,3}$ Some estimates of local recurrence reach $60 \%$ by 10 years. With further follow-up, local recurrence rates of $\sim 70 \%$ at 15 years are appreciated. $^{2}$ The risk of local recurrence does not appear to plateau. Death from sarcoma within the first 5 years of primary WD-LPS resection is unlikely but not rare. Disease-specific survival at 5 years was $83 \%$ in the 99 patients managed at MSKCC. Nevertheless, over time centrally located deposits of recurrent LPS and repeated attempts at resection constitute a threat to life. After a few experiences with rapid recurrence before the resolution of significant postoperative complications, the surgeon is apt to become reflective and seek guidance regarding patient selection for salvage surgery. ${ }^{4}$

Patient selection for salvage surgery in recurrent RPS. When recurrent RPS is considered in general, the likelihood of prolonged disease-free and/or overall survival following salvage surgery is predicted by a variety of factors that can be viewed as surrogates for the "biology" 
of the individual patient's tumor. In a group of patients with local relapse after primary resection, disease-free interval (DFI, from primary resection to the detection of recurrence) predicted OS. ${ }^{5}$ In primary and recurrent RPS, multifocality predicts poor OS. ${ }^{6}$ Although these analyses were not histologic subtype specific, it is very likely that these variables apply most aptly to liposarcoma, because this is the most frequent histology overall in the retroperitoneum, has the highest rate of local relapse, and the greatest likelihood that patients would be considered for salvage surgery for local recurrence. The latter is particularly true for WD-LPS with its usually indolent behaviour and preserved patient performance status.

The most often quoted work about patient selection for surgery for recurrent RP LPS was published by Singer and colleagues in $2009 .{ }^{7}$ In their experience of 61 salvage resections performed from 1982 through 2005, these authors noted a significantly inferior outcome in patients with faster-growing tumors. The cut off was $0.9 \mathrm{~cm} / \mathrm{month}$, popularly rounded off to yield the $1 \mathrm{~cm} /$ month rule. The patient cohort in that report included both WD-LPS $(n=48)$ and DD-LPS $(n=49)$. The validity of the rule was not examined in each subtype, and one might assume that the faster-growing tumors were dedifferentiated. Thus, the paper by Ikoma et al. adds a new dimension, focusing exclusively on WD-LPS. ${ }^{1}$

This singular focus on recurrent WD-LPS of the retroperitoneum is noteworthy, and the information provided is immensely useful. This is the histologic subtype for which considerations about risk/benefit of salvage surgery is the most relevant, because these patients have the most extended survival, albeit with an extremely local high relapse rate following resection of first local recurrence (84\% at a median follow-up time of 4.5 years in this series of 45 patients with an $R_{0} / R_{1}$ resection). In the experience at MDACC, DFI $<1$ year and organ invasion at primary resection predicted inferior OS. As the authors note, these variables, together with multifocality, are measures of biologic aggressiveness. Of even greater interest, resection within 6 months of detection of first recurrence portended poor DFS, implying that a period of observation before making the decision for salvage surgery may prevent precipitous surgery for patients who are destined to develop rapid, multifocal recurrence due to innate biologic aggressiveness. The experience of the MDACC group reinforces the importance of considering tumor biology, even within the envelope of WD-LPS.

Some missing pieces of information would help to frame the MDACC results in a wider context. The authors have not described how their patients were selected for resection or from a field of how many. Data for patients who have been referred for consideration of resection, but did not reach the operating theatre, are notoriously difficult to find. Population-based data would be highly valuable, but few jurisdictions keep detailed data on recurrent cancer and its outcomes. Registry data could provide the "denominator" from which the patients who are taken for resection are eventually selected, but to be as unbiased as possible, the registry must include all patients with recurrence, not just those referred to a specialized center. The real denominator is clearly extremely challenging to capture. One partial solution is to track all patients who undergo resection of primary disease at a given institution(s) within a specific timeframe and then report the outcomes of all those who develop recurrence. The results of such an exercise are sobering. The TARPSWG collaborative recently reported recurrence patterns and outcomes following relapse, starting with a group of 1007 consecutive patients who had primary RPS resection at 1 of 8 specialized sarcoma centres between January 2002 and December 2011. ${ }^{8}$ A total of 263 of the 1007 patients had WD-LPS. In the group of 408 patients who recurred after $R_{0} / R_{1}$ of primary RPS, the site of relapse was local only at the time of first detection in 219, and in this subgroup, the subsequent 5 -year OS was $29 \%$. In the patients with local-only disease who had resection $(n=105)$, the rate of local rerecurrence at 5 years was $58 \%$, and the estimated 5 -year OS was $43 \%$.

The TARPSWG collaborative has recently specifically examined the outcomes after resection of first local recurrence of RPS at 22 participating sarcoma centers, between January 2002 and December 2011 (Raut et al., personal communication). Of a total of 602 patients in the entire cohort, 169 had WD-LPS. In the latter group, 5-year crude cumulative incidence of local recurrence was $55 \%$, of distant recurrence 5\%, and 5-year DFS and OS were 36 and $82 \%$, respectively, following resection of first local recurrence. Based on their pooled data, the collaborative has developed a nomogram for predicting outcomes in recurrent RPS, with publication expected in 2018.

Other treatment options. Given the poor outcomes in the subgroup of patients who undergo resection of recurrent WD-LPS despite indicators of adverse biology, one must consider what other treatment options we can offer such patients. ${ }^{1}$ There is ongoing controversy about the sensitivity of WD-LPS to radiation treatment, and many centers that employ preoperative XRT in the management of primary RPS would not consider WD-LPS to be an indication for this treatment. The radiation-associated toxicity that ensues when radiation is used in the postoperative setting 
will be encountered when patients with local recurrence are treated with XRT, narrowing the potential therapeutic window. For these reasons, we tend to reserve XRT for patients who have significant symptoms and no other treatment options.

Systemic therapy is an option for patients with recurrent WD-LPS whose tumor has been deemed unresectable, although there is a relative paucity of subtype-specific data to guide management in this scenario. While the objective response rate with conventional doxorubicin-based therapy is low (10-15\% at best), the clinical benefit rate may be somewhat higher. ${ }^{9,10}$ A more targeted approach, such as mdm2 inhibition, has a strong rationale, but this promise has yet to be borne out in the clinical setting. In a group of 37 patients with unresectable and/or metastatic WD-LPS, largely of retroperitoneal origin, median OS was 33.5 months, and 5-year OS was $\sim 20 \%$. These data provide a striking reminder of the eventual fatality of this disease.

Finally, I would like to make a plea for further study and implementation of validated quality of life measures in patients with retroperitoneal sarcoma, as well as decision aids. Nowhere is this more relevant than in developing a management plan for the patient with recurrent WD-LPS who is currently feeling well and may live for years without resection. Nomograms can help us to predict hard outcomes and are definitely valuable in highlighting the limitations of resection. However, we have not made sufficient efforts to study physical and emotional well-being in patients who live with recurrent RPS or experience the sometimes-dire complications of attempted resection.

\section{REFERENCES}

1. Ikoma N, Roland CL, Torres KE, et al. Salvage surgery for recurrent retroperitoneal well-differentiated liposarcoma: early reoperation may not provide benefit. Ann Surg Oncol. 2018. https://doi.org/10.1245/s10434-018-6417-6.

2. Singer S, Antonescu CR, Riedel E, Brennan MF. Histologic subtype and margin of resection predict pattern of recurrence and survival for retroperitoneal liposarcoma. Ann Surg. 2003;238:358-71.

3. Gronchi A, Strauss DC, Miceli R, et al. Variability in patterns of recurrence after resection of primary retroperitoneal sarcoma (RPS): a report on 1007 patients from the Multi-institutional Collaborative RPS Working Group. Ann Surg. 2016;263:1002-9.

4. Hamilton TD, Cannell AJ, Kim M, et al. Results of resection for recurrent or residual retroperitoneal sarcoma after failed primary treatment. Ann Surg Oncol. 2017;24:211-8.

5. Gronchi A, Miceli R, Allard MA, et al. Personalizing the approach to retroperitoneal soft tissue sarcoma: histology-specific patterns of failure and postrelapse outcome after primary extended resection. Ann Surg Oncol. 2015;22:1447-54.

6. Anaya DA, Lahat G, Liu J, et al. Multifocality in retroperitoneal sarcoma: a prognostic factor critical to surgical decision-making. Ann Surg. 2009;249:137-42.

7. Park JO, Qin LX, Prete FP, et al. Predicting outcome by growth rate of locally recurrent retroperitoneal liposarcoma: the one centimeter per month rule. Ann Surg. 2009;250:977-82.

8. MacNeill AJ, Miceli R, Strauss DC, et al. Post-relapse outcomes after primary extended resection of retroperitoneal sarcoma: a report from the Trans-Atlantic RPS Working Group. Cancer. 2017;123:1971-8.

9. Italiano A, Toulmonde M, Cioffi A, et al. Advanced well-differentiated/dedifferentiated liposarcomas: role of chemotherapy and survival. Ann Oncol. 2012;23:1601-7.

10. Jones RL, Fisher C, Al-Muderis O, Judson IR, et al. Differential sensitivity of liposarcoma subtypes to chemotherapy. Eur $J$ Cancer. 2005;41:2853-60. 